\title{
ON THE ASYMPTOTIC BEHAVIOR OF LINEAR SYSTEMS
}

\section{PHIL LOCKE}

Abstract. The purpose of this paper is to establish a necessary and sufficient condition for the vector-matrix system $\dot{x}=[A(t)+$ $B(t)] x$ to have solutions of the form $Y(t) c(t)$ where $Y(t)$ is a fundamental matrix of solutions of $\dot{y}=A(t) y$.

Consider the linear systems

$$
\begin{aligned}
& \dot{y}=A(t) y, \\
& \dot{x}=[A(t)+B(t)] x,
\end{aligned}
$$

where $x, y$ are $n$-vectors and $A(t), B(t)$ are continuous $n \times n$ matrices on $\left[t_{0}, \infty\right)$. Let $Y(t)$ be a fundamental matrix of solutions of (1) and put $Z(t)=Y^{-1}(t) B(t) Y(t)$. Let \|\| denote any appropriate vectormatrix norm. We establish the following result.

THEOREM. Given an arbitrary constant n-vector c, equation (2) has a unique solution $x(t)$ of the form

$$
x(t)=Y(t) c(t),
$$

where $c(t)$ is an n-vector function satisfying

$$
\lim _{t \rightarrow \infty} c(t)=c, \quad \int_{t_{0}}^{\infty}\|\dot{c}(t)\| d t<\infty
$$

if and only if

$$
\int_{t_{0}}^{\infty}\|Z(t)\| d t<\infty
$$

REMARK. The sufficiency of condition (5) has been established by Bebernes and Vinh [1]. (Bebernes and Vinh do not mention that $\int_{t_{0}}^{\infty}\|\dot{c}(t)\| d t<\infty$, but it follows readily from their proof.) Our proof of the sufficiency of condition (5), while similar to theirs, differs in several significant details and is therefore included below.

Proof. First assume that (5) holds. Let $c$ be a given $n$-vector. Let $X(t)$ be a fundamental matrix solution of (2) and let the matrix $C(t)$ be defined by the "variation of parameter" equation $X(t)=Y(t) C(t)$. It is easily shown that $C(t)$ satisfies

Received by the editors September 11, 1969.

A MS Subject Classifications. Primary 3420, 3450.

Key Words and Phrases. Asymptotic behavior, linear system, fundamental matrix, variation of parameter, Gronwall inequality, Liouville's theorem, Wronskian. 


$$
\dot{C}(t)=Z(t) C(t) .
$$

Integrating and applying Gronwall's inequality, it follows that $\|C(t)\|$ is bounded on $\left[t_{0}, \infty\right)$. Hence (5) and (6) imply that $\int_{t_{0}}^{\infty}\|\dot{C}(t)\| d t<\infty$. Hence $\int_{t_{0}}^{\infty} \dot{C}(t) d t$ converges, whence $\lim _{t \rightarrow \infty} C(t)$ exists. Denote this limit by $C$. By Liouville's theorem

$$
\operatorname{det} C(t)=\operatorname{det} C\left(t_{0}\right) \exp \int_{t_{0}}^{t} \operatorname{trace} Z(s) d s .
$$

Certainly det $C\left(t_{0}\right) \neq 0$; by (5) we can let $t \rightarrow \infty$, concluding that $\operatorname{det} C$ $\neq 0$. Take $c(t)=C(t) C^{-1} c, x(t)=X(t) C^{-1} c$. The uniqueness of $c(t)$ is readily established.

Conversely, suppose that given any $n$-vector $c,(2)$ has a (unique) solution $x(t)$ of the form (3) satisfying (4). Thus, in particular, given a nonsingular square matrix $C,(2)$ has a (fundamental) matrix solution $X(t)$ of the form $X(t)=Y(t) C(t)$ where $C(t)$ satisfies $\lim _{t \rightarrow \infty} C(t)=C$, $\int_{t_{0}}^{\infty}\|\dot{C}(t)\| d t<\infty$. Since $C(t)$ must satisfy $(6)$ and since $\lim _{t \rightarrow \infty} \operatorname{det} C(t)$ $=\operatorname{det} C \neq 0$, it follows that $\operatorname{det} C(t) \neq 0$ on $\left[t_{0}, \infty\right)$. Thus $\lim _{t \rightarrow \infty} C^{-1}(t)$ $=C^{-1}$, whence $\left\|C^{-1}(t)\right\|$ is bounded on $\left[t_{0}, \infty\right)$. Since $Z(t)=\dot{C}(t) C^{-1}(t)$, it follows that condition (5) holds. This completes the proof.

Related results for scalar equations may be obtained as corollaries. In particular, consider the $n$ th-order linear scalar equations

$$
\begin{aligned}
& v^{(n)}=\sum_{j=0}^{n-1} a_{j}(t) v^{(j)}, \\
& u^{(n)}=\sum_{j=0}^{n-1}\left[a_{j}(t)+b_{j}(t)\right] u^{(j)},
\end{aligned}
$$

where the coefficient functions are continuous on $\left[t_{0}, \infty\right)$. Let $v_{1}(t)$, $\cdots, v_{n}(t)$ be a fundamental set of solutions of $\left(1^{*}\right)$. Let $W(t)$ denote their Wronskian. Let $W_{k}(t)(k=1, \cdots, n)$ be the determinant obtained by replacing all elements in the $k$ th column of $W(t)$ by zero except the element in the $n$th row which is replaced by 1 . Let

$$
M(t)=\max _{1 \leqq k \leqq n}\left|\sum_{j=0}^{n-1} b_{j}(t) v_{k}^{(j)}(t)\right| .
$$

We have the following result.

Corollary. Assume

$$
\int_{t_{0}}^{\infty}\left|a_{n-1}(t)\right| d t<\infty .
$$


Given arbitrary constants $c_{1}, \cdots, c_{n}$, equation (2*) has a unique solution $u(t)$ of the form

$$
u^{(j)}(t)=\sum_{k=1}^{n} c_{k}(t) v_{k}^{(j)}(t), \quad j=0, \cdots, n-1,
$$

satisfying

$$
\lim _{t \rightarrow \infty} c_{k}(t)=c_{k}, \quad \int_{t_{0}}^{\infty}\left|\dot{c}_{k}(t)\right| d t<\infty
$$

if and only if

$$
\int_{t_{0}}^{\infty}\left|W_{k}(t)\right| M(t) d t<\infty, \quad k=1, \cdots, n .
$$

Proof. Equations $\left(1^{*}\right)$ and $\left(2^{*}\right)$ are easily seen to be equivalent, respectively, to linear systems of the form (1) and (2) with

$$
\begin{aligned}
A(t)= & {\left[\begin{array}{cccc}
0 & 1 & \cdots & 0 \\
0 & 0 & \cdots & 0 \\
\cdot & \vdots & & \vdots \\
\dot{0} & 0 & \cdots & \dot{1} \\
a_{0}(t) & a_{1}(t) & \cdots & a_{n-1}(t)
\end{array}\right], } \\
B(t)= & {\left[\begin{array}{cccc}
0 & 0 & \cdots & 0 \\
0 & 0 & \cdots & 0 \\
\cdot & \cdot & & : \\
\cdot & \cdot & & \cdot \\
b_{0}(t) & b_{1}(t) & \cdots & b_{n-1}(t)
\end{array}\right] . }
\end{aligned}
$$

Condition (5) is found to be equivalent to $\int_{t_{0}}^{\infty}\left(\left|W_{k}(t)\right| /|W(t)|\right) M(t) d t$ $<\infty, k=1, \cdots, n$. Liouville's theorem, together with (7), implies that the above condition is equivalent to $\left(5^{*}\right)$.

REMARK. The above corollary sharpens a result of Katz [2] who assumes that $a_{n-1}(t) \equiv b_{n-1}(t) \equiv 0$ on $\left[t_{0}, \infty\right)$, a stronger requirement than (7).

\section{REFERENCES}

1. J. W. Bebernes and N. X. Vinh, On the asympotic behavior of linear differential equations, Amer. Math. Monthly 72 (1965), 285-287. MR 31 \#6011.

2. I. N. Katz, Asymptotic behavior of solutions to some nth order linear differential equations, Proc. Amer. Math. Soc. 21 (1969), 657-662.

University of Maine, Orono, Maine 04473 